\title{
The perpetual enigma of bronchoalveolar lavage fluid lymphocytosis in chronic hypersensitivity pneumonitis: is it of diagnostic value?
}

\author{
Francesco Bonella (D) and Ulrich Costabel
}

Affiliation: Center for Interstitial and Rare Lung Diseases, Pneumology Department, Ruhrlandklinik University Hospital, University of Duisburg-Essen, Essen, Germany.

Correspondence: Francesco Bonella, Ruhrlandklinik University Hospital, Tüschener Weg 4045239 Essen, Germany. E-mail: Francesco.bonellaarlk.uk-essen.de

@ERSpublications

A marked increase in BAL lymphocyte percentage is of additional value in distinguishing cHP from other forms of fibrotic ILD https://bit.ly/3ciR8zG

Cite this article as: Bonella F, Costabel U. The perpetual enigma of bronchoalveolar lavage fluid lymphocytosis in chronic hypersensitivity pneumonitis: is it of diagnostic value? Eur Respir J 2020; 56: 2001534 [https://doi.org/10.1183/13993003.01534-2020].

Since its introduction in the 1970s, bronchoalveolar lavage (BAL) has been increasingly recognised as a low-risk investigational tool, providing information of diagnostic and, potentially, prognostic value in several fields of pulmonary medicine, especially in interstitial lung disease (ILD) [1]. In some ultrarare ILDs, such as pulmonary alveolar proteinosis, BAL findings are highly specific and diagnostic, whereas in the more common ILDs they are nonspecific and cannot provide a confident diagnosis as a standalone test. However, when BAL cellular analysis is interpreted in the context of clinical and high-resolution computed tomography (HRCT) findings and in an experienced multidisciplinary discussion (MDD) setting, it may contribute to narrowing the differential diagnosis and help to avoid surgical lung biopsy.

What did ILD guidelines of the past two decades tell us? In the American Thoracic Society (ATS)/ European Respiratory Society (ERS) statement on idiopathic pulmonary fibrosis (IPF) of 2000 [2], BAL was considered one of the requirements for the exclusion of other diseases in a patient with IPF who did not undergo surgical lung biopsy, and was included as one of the four major criteria for making a clinical diagnosis of IPF. BAL was also found to be useful in the diagnostic work-up of ILDs in the ATS/ERS international consensus classification of idiopathic interstitial pneumonias (IIPs) of 2002 [3]. In the first evidence-based international IPF guidelines of 2011, however, there was a weak negative recommendation against performing a BAL cellular analysis in the diagnostic evaluation of suspected IPF [4]. The revival occurred in 2018, when BAL cellular analysis received a conditional recommendation in the ATS/ERS/ Japanese Respiratory Society/Latin American Thoracic Association clinical practice guideline on IPF diagnosis for those patients with an HRCT pattern of probable usual interstitial pneumonia (UIP), indeterminate or alternative diagnosis [5]. The guideline mentioned as desirable consequences that BAL may help to distinguish IPF from some other ILDs, most notably eosinophilic pneumonia, cryptogenic organising pneumonia, sarcoidosis, or infections. Interestingly, and much to our surprise, hypersensitivity pneumonitis (HP) is missing in this list, due to the fact that only two studies were included in the meta-analysis of BAL cellular analysis in that guideline. 
What is the reason of this fluctuating perception of the role of BAL in the diagnostic approach to IPF? The most challenging differential diagnosis of IPF is indeed chronic hypersensitivity pneumonitis (cHP). The characteristic BAL finding of HP is a marked lymphocytosis (usually $>50 \%$ ), which is considered to be less pronounced in chronic fibrotic HP [6-9], but is usually at a higher level compared with other ILDs $[10,11]$. In this context, it has been suggested that BAL lymphocytosis of $>30 \%$ discriminates chronic fibrotic HP with a UIP pattern on HRCT from IPF [12]. In addition, neutrophils, eosinophils, mast cells and, more characteristically, plasma cells may be mildly elevated $[6,7,13]$. Morphological BAL cellular features, like T cell activation and foamy macrophages, can also be useful to support HP diagnosis [14]. The clinical utility of a decreased CD4/CD8 ratio, ranging typically between 0.5 and 1.5 in HP, is under debate because it is highly variable and frequently not decreased but even elevated in cHP $[15,16]$

What is the best BAL lymphocyte threshold to discriminate IPF from cHP in the clinical routine? Apart from older studies demonstrating that the absence of BAL lymphocytosis is important for the diagnosis of IPF [17, 18], Онsнiмо et al. [12] were the first to explore the discriminative power of cut-off levels for lymphocytosis in BAL and found that a cut-off level of $<30 \%$ for lymphocytes in BAL had a favourable discriminative power for the diagnosis of IPF. In a recent Delphi survey involving 45 ILD experts from 14 countries, the vast majority of the experts rated BAL lymphocytosis $>40 \%$ as "important/very important" for the diagnosis of cHP, while levels between 30 and $39 \%$ were considered a grey zone, and values between 20 and $30 \%$ as unhelpful [19]. TzILAS et al. [20] reported that a BAL lymphocytosis of $>20 \%$ was of added diagnostic value in a retrospective cohort of undiagnosed fibrotic ILD with an indeterminate for UIP pattern on HRCT, changing the diagnosis from IPF to HP in $15 \%$ of the cases. Summarising the evidence, the current situation is paradoxical: BAL lymphocytosis is widely used in establishing a diagnosis of cHP and to discriminate it from IPF, but its precise diagnostic role and best cut-off value remains to be determined, since no studies have robustly tested the additive discriminative value of BAL lymphocytosis in differentiating $\mathrm{cHP}$ from other forms of fibrotic ILD in the past.

In an article now published in the European Respiratory Journal, ADDERLEy et al. [21] shape things up by reporting the results of a systematic review (53 studies) and meta-analysis (42 studies) on this topic. They were also able to obtain individual patient level data from eight of the studies to analyse the performance characteristics of BAL lymphocyte thresholds. Interestingly, one third of the studies originated from Japan, and only about half included comparator populations of non-cHP ILD, most frequently of IPF followed by sarcoidosis. As a first main finding of the meta-analysis, the authors showed that the pooled estimate for BAL lymphocyte percentage in cHP was $43 \%$ and higher compared with all other ILDs, with statistically significant differences compared with IPF and with non-IPF IIPs, but only numerically higher in cHP compared to connective tissue disease-associated ILD (CTD-ILD) and sarcoidosis. Interestingly, a sensitivity analysis using 26 studies that defined cHP based on signs of fibrosis on HRCT and/or biopsy provided a similar pooled estimate of $44 \%$ for BAL lymphocytes. This clearly demonstrates that even fibrotic cHP is characterised by a pronounced BAL lymphocytosis, similar to non-fibrotic cHP or acute HP. However, considering the high level of heterogeneity of the included studies, especially in the clinical-radiological criteria to define cHP, the results should be taken with caution. Under a different perspective, and considering the limited numbers of BAL studies in CTD-ILDs [22], with many of them combining different rheumatological diseases, this metanalysis confirms that lymphocytosis (around 23\%) is the predominant finding in BAL of CTD-ILD but, unfortunately, without discriminative value against non-IPF IIPs (also around 23\%).

More complicated is the interpretation of the performance analysis of BAL lymphocyte thresholds to discriminate cHP from other ILDs. The identification of a threshold that provides best sensitivity and specificity suggests that such a value could be tested prospectively to determine the performance of BAL cellular analysis as a diagnostic test in cHP. To address this question, the authors chose to use individual patient level data from eight studies, pooled as a single cohort. They show that a lymphocytosis of $>20 \%$ in BAL would be suitable to consider $\mathrm{cHP}$ as a diagnosis, with a sensitivity of $68 \%$ and a specificity of $65 \%$ for discriminating cHP from other ILDs and IPF. In front of a low positive predictive value (PPV) of only $41 \%$, this threshold gives a negative predictive value of $85 \%$. Generally speaking, such a performance cannot be considered as sufficient for a diagnostic test, which should be routinely adopted either to rule in or out a disease. Comparing BAL with HRCT for the diagnosis of IPF, in front of the same low sensitivity (40 to 80\%), the PPV of a UIP pattern on HRCT is much higher $(>90 \%)$ [5]. As the authors correctly discuss, higher thresholds of BAL lymphocytosis would provide greater specificity at the cost of a lower sensitivity and could be more useful in the clinical practice.

Noteworthy ancillary results of a multivariate analysis showed that older age and a history of smoking, but not antigen type, forced vital capacity or diffusing capacity of the lung for carbon monoxide were associated with a lower BAL lymphocyte percentage in patients with cHP. Interestingly, DE SADELEER et al. [23], who used a similar cut-off of $20 \%$ for BAL lymphocytosis in their study of $91 \mathrm{cHP}$ patients with fibrosis, showed that low lymphocyte percentage was associated with poor 10-year survival (HR 2.66, 95\% CI 1.05-6.73; $\mathrm{p}=0.038$ ) and with the presence of honeycombing on HRCT, whereas no differences in 
functional disease severity, at time of BAL, were seen between patients with low and high BAL lymphocytes. Further studies to address the prognostic aspect of BAL lymphocyte proportions and the association with parameters of disease severity are clearly needed.

Overall, the well-performed meta-analysis addresses an important clinical issue and provides some guidance for the use of BAL in the diagnostic assessment of cHP. The data strongly suggest that a marked increase in BAL lymphocyte percentage is of additional value in distinguishing cHP from other fibrotic ILDs. The authors are careful to describe the limitations of their study, especially the high heterogeneity of the included studies, their mostly retrospective nature and low data quality and, above all, the exclusion of acute/non-fibrotic forms of HP from the initial search. Is there light at the end of the tunnel? The answer is in the conclusion of this paper. Further work is needed to extend and refine this meta-analysis, not only in reducing the above-mentioned incorporation biases, but also by testing the discriminative performance of lymphocyte percentage in established diagnostic prediction models (do such models exist?). In order to optimise interpretation of BAL data, we also need to better understand the relationships between BAL lymphocytosis, antigen exposure and host factors in cHP. But a doubt remains: are our expectations towards BAL lymphocytosis too high?

Conflict of interest: F. Bonella reports personal fees and non-financial support from Boehringer Ingelheim, Roche, BMS, Galapagos and Savara, outside the submitted work. U. Costabel has nothing to disclose.

\section{References}

1 Bonella F, Ohshimo S, Bauer P, et al. Bronchoalveolar lavage. In: Strausz J, Bolliger CT, eds. Interventional Pulmonology (ERS Monograph). Sheffield, European Respiratory Society, 2010; pp. 59-72.

2 American Thoracic Society. Idiopathic pulmonary fibrosis: diagnosis and treatment. International consensus statement. American Thoracic Society (ATS), and the European Respiratory Society (ERS). Am J Respir Crit Care Med 2000; 161: 646-664.

3 American Thoracic Society/European Respiratory Society international multidisciplinary consensus classification of the idiopathic interstitial pneumonias. Am J Respir Crit Care Med 2002; 165: 277-304.

4 Raghu G, Collard HR, Egan JJ, et al. An official ATS/ERS/JRS/ALAT statement: idiopathic pulmonary fibrosis: evidence-based guidelines for diagnosis and management. Am J Respir Crit Care Med 2011; 183: 788-824.

5 Raghu G, Remy-Jardin M, Myers JL, et al. Diagnosis of idiopathic pulmonary fibrosis. an official ATS/ERS/JRS/ ALAT clinical practice guideline. Am J Respir Crit Care Med 2018; 198: e44-e68.

$6 \quad$ Costabel U. The alveolitis of hypersensitivity pneumonitis. Eur Respir J 1988; 1: 5-9.

7 Semenzato G, Bjermer L, Costabel U, et al. Clinical guidelines and indications for bronchoalveolar lavage (BAL): extrinsic allergic alveolitis. Eur Respir J 1990; 3: 945-946.

8 Ohtani Y, Saiki S, Kitaichi M, et al. Chronic bird fancier's lung: histopathological and clinical correlation. An application of the 2002 ATS/ERS consensus classification of the idiopathic interstitial pneumonias. Thorax 2005; 60: 665-671.

9 Gaxiola M, Buendia-Roldan I, Mejia M, et al. Morphologic diversity of chronic pigeon breeder's disease: clinical features and survival. Respir Med 2011; 105: 608-614.

10 Haslam PL, Dewar A, Butchers P, et al. Mast cells, atypical lymphocytes, and neutrophils in bronchoalveolar lavage in extrinsic allergic alveolitis. Comparison with other interstitial lung diseases. Am Rev Respir Dis 1987; 135: 35-47.

11 Schildge J, Nagel C, Grun C. Bronchoalveolar lavage in interstitial lung diseases: does the recovery rate affect the results? Respiration 2007; 74: 553-557.

12 Ohshimo S, Bonella F, Cui A, et al. Significance of bronchoalveolar lavage for the diagnosis of idiopathic pulmonary fibrosis. Am J Respir Crit Care Med 2009; 179: 1043-1047.

13 Drent M, Wagenaar S, van Velzen-Blad H, et al. Relationship between plasma cell levels and profile of bronchoalveolar lavage fluid in patients with subacute extrinsic allergic alveolitis. Thorax 1993; 48: 835-839.

14 Costabel $\mathrm{U}$, Bross $\mathrm{KJ}$, Ruhle $\mathrm{KH}$, et al. Ia-like antigens on T-cells and their subpopulations in pulmonary sarcoidosis and in hypersensitivity pneumonitis. Analysis of bronchoalveolar and blood lymphocytes. Am Rev Respir Dis 1985; 131: 337-342.

15 Ando M, Konishi K, Yoneda R, et al. Difference in the phenotypes of bronchoalveolar lavage lymphocytes in patients with summer-type hypersensitivity pneumonitis, farmer's lung, ventilation pneumonitis, and bird fancier's lung: report of a nationwide epidemiologic study in Japan. J Allergy Clin Immunol 1991; 87: 1002-1009.

16 Barrera L, Mendoza F, Zuniga J, et al. Functional diversity of T-cell subpopulations in subacute and chronic hypersensitivity pneumonitis. Am J Respir Crit Care Med 2008; 177: 44-55.

17 Nagai S, Handa T, Ito Y, et al. Bronchoalveolar lavage in idiopathic interstitial lung diseases. Semin Respir Crit Care Med 2007; 28: 496-503.

18 Nagao T, Nagai S, Kitaichi M, et al. Usual interstitial pneumonia: idiopathic pulmonary fibrosis versus collagen vascular diseases. Respiration 2001; 68: 151-159.

19 Morisset J, Johannson KA, Jones KD, et al. Identification of diagnostic criteria for chronic hypersensitivity pneumonitis: an international modified Delphi survey. Am J Respir Crit Care Med 2018; 197: 1036-1044.

20 Tzilas V, Tzouvelekis A, Bouros E, et al. Diagnostic value of BAL lymphocytosis in patients with indeterminate for usual interstitial pneumonia imaging pattern. Eur Respir J 2019; 54: 1901144.

21 Adderley N, Humphreys CJ, Barnes $\mathrm{H}$, et al. Bronchoalveolar lavage fluid lymphocytosis in chronic hypersensitivity pneumonitis: a systematic review and meta-analysis. Eur Respir J 2020; 56: 2000206.

22 Costabel U, Guzman J, Bonella F, et al. Bronchoalveolar lavage in other interstitial lung diseases. Semin Respir Crit Care Med 2007; 28: 514-524.

23 De Sadeleer LJ, Hermans F, De Dycker E, et al. Impact of BAL lymphocytosis and presence of honeycombing on corticosteroid treatment effect in fibrotic hypersensitivity pneumonitis: a retrospective cohort study. Eur Respir J 2020; 55: 1901983. 